\title{
Survey on Tea Discriminator
}

\author{
Princee Gupta \\ M. Tech. Scholar \\ SIRT, Bhopal
}

\author{
Rajesh K.Shukla \\ HOD, CSE \\ SIRT, Bhopal
}

\begin{abstract}
A number of beverages viz. hard drink, soft drink, packet juice, tea are being used around the globe having different chemical compositions. Organoleptic Systems are being used for a variety of detection tasks from checking quality of food products to medicinal diagnosis by detecting chemical compositions. The entire organoleptic system, from sample delivery stage to classification stage, is usually optimized to a particular problem domain in order to provide suitable sensing performance. The optimization of sample preparation, signal processing, feature extraction, classifier are as important as choice of sensors within the array in enhancing the performance of the organoleptic system because presence of irrelevant features increases the dimensionality of the search space, which can do reverse effect on the accuracy of the Pattern Recognition (PARC) techniques.

Tea in present world is the most popular beverage having enormous global marketing. Various researchers have made a number of efforts to correlate tea quality with its chemical composition which led to many humanoid errors and may vary from person to person. This problem can be solved by using an instrument called "Electronic Tongue (i-tongue)". It is developed to reduce human sensory test panels, get accurate measurement of taste, prepare optimized development time and cost. This system analyses liquid including an array of non-specific chemical sensors with partial specificity for different component in liquid samples and appropriate pattern recognition capable of recognizing the qualitative and quantitative composition of sample and complex solutions. A number of attempts have been performed using sensor array and electrochemical techniques such as Cyclic Voltammetry, Potentiometry and Conductivity to classify different types of tea.
\end{abstract}

\section{Keywords}

PARC, Electronic Tongue, i-tongue, Pattern Recognition, Potentiometry, Voltammetry, Conductivity.

\section{INTRODUCTION}

Instrumental evaluation [1] of quality of black tea is quite complicated because of the presence of immeasurable compounds and their multidimensional influence in determining the final quality of tea. Many experienced tea tasters are employed for gradation of tea on the basis of their scores [2], this method is subjective and a low-cost equipment is desired in the tea industry.

CSIO, Chandigarh by Agrionics Group developed an Electronic-Tongue [3] that employs nonspecific multielectrode electrochemical impedance spectroscopy for classification of Indian black tea. An i-tongue employs electrochemical impedance spectroscopy which is a powerful and general technique suitable for characterization of electrochemical systems on the basis of impedance reading being collected at particular frequency interval and could also distinguish different substances eliciting the same basic taste [4]. For evaluation of black tea quality using electronic tongue, pioneering work had been done, where the efficiency of the electronic tongue instrument in classifying black tea in different processing stages was established. The impedance response at logarithmic frequency interval in the predefined range is used by one of the feature extraction method like PCA which reduces the dimensions and before this feature extraction, the features are being selected by one of the feature selection method to select the best feature in the dataset provided. Feature selection techniques [5] are based on the design of a standard function and the selection of a search strategy. There are many feature selection techniques viz. genetic Algorithm, Particle Swarm Optimization, Ant Colony Optimization, simulated Annealing are employed as search strategies.

The i-tongue has been successfully employed for recognition. Classification, and quality analysis of various food, beverages and agro products, viz., wine, cola, meat, fish, coffee, etc. Also, it has successfully established its capability for other applications like medical diagnostics, environment monitoring and legal protection of inventions-digital fingerprints of taste and odors.

\subsection{Benefits of i-tongue:}

- It estimates and enumerates bitterness scores of new chemical entities (NCE) [6].

- It Optimizes and escalations the formulation development process.

- It measures the efficiency of complexion/coating Within the formulation.

- It tests various combinations of sweeteners, enhancers, exhausters, aromas and masking agents in less time.

- It compares the deliciousness of new formulations with competitor's products.

- It serves a quality control function for flavored products and excipients.

- It cultivates suitable matching bitter placebo for double blind clinical testing.

- It defines consistency of organoleptic quality of small production batches to full-scale manufacturing.

\subsection{Classifications:}

Supervised learning or Associative learning in which the network is trained by providing it with input and matching output patterns. These input-output pairs can be provided by an external teacher, or by the system which contains the neural network (self-supervised).

Unsupervised learning or Self-organization in which an (output) unit is trained to respond to clusters of pattern within the input. In this paradigm the system is supposed to discover 
statistically salient features of the input population. Unlike the supervised learning paradigm, there is no a priori set of categories into which the patterns are to be classified; rather the system must develop its own representation of the input stimuli.

Reinforcement Learning may be considered as an intermediate form of the above two types of learning. Here the learning machine does some action on the environment and gets a feedback response from the environment. The learning system grades its action good (rewarding) or bad (punishable) based on the environmental response and accordingly adjusts its parameters. Generally, parameter adjustment is continued until an equilibrium state occurs, following which there will be no more changes in its parameters. The self-organizing neural learning may be categorized under this type of learning.

\section{PRELIMINARY OF SVM}

Tea is one of the widely consumed beverage in the world and India being the second largest manufacturer, has its tremendous agro-commercial importance. A number of efforts have been made to classify different tea using sensor array and electrochemical techniques such as Cyclic Voltammetry, Potentiometry and Conductivity. However, in comparison to potentiometry, especially with voltammetry, the impedance measurements are advantageous because of the potential experimental simplicity and the reduction of the response times [7].

Instrumental evaluation of black tea is quite complex because of presence of many compounds and therefore it is being distinguished by tea tasters on their scores $[1,8]$. Impedance tongue are sensor array for qualitative and quantitative analysis and it is used to differentiate basic standard taste. The classification models can be created by using supervised and unsupervised techniques and artificial neural network $[9,10]$.

Phaisangittisagul [11] proposed an approach of sensor subset selection in machine olfaction in which each sensor should provide different selectivity profiles over the range of target odor application so that a unique odor pattern is produced from each sensor in the array. He employed, Support Vector Machine (SVM) as a classification algorithm, from the experimental results on the soda data set, the numbers of selected sensors were not only significantly reduced but the classification performance was also increased.

Tan et al. [12] used Support Vector Machines (SVM) technique to identify fuel types. Flame oscillation signal were captured by a three-cell flame monitor. Thirty flame features were extracted from each flame signal. Then Principal Component Analysis (PCA) was used to choose the principal components of each features vector. An SVM was deployed to map the principal components, size-reduced flame features, to an individual type of fuel. The data of eight different types of coal obtained from a combustion test facility demonstrated that the SVM technique was effective for identifying the fuel types

Wang et al. [13] presented a new intelligent method for signals recognition of electronic nose, based on support vector machine (SVM) classification. The SVM operates on the principle of structure risk minimization hence a better generalization ability is guaranteed. The experiments of the recognition of three different gases, ethanol, gasoline and acetone, have been presented and the method achieves higher recognition rate at reasonably small size of training sample set.

Zheng et al. [14] review some pattern recognition schemes published in recent years. After giving the general processing steps of pattern recognition, they discuss several methods used for steps of pattern recognition such as Principal Component Analysis (PCA) in feature extraction, Support Vector Machines (SVM) in classification, and so forth. Different kinds of merits are presented and their applications on pattern precognition are given.

\section{PATTERN RECOGNITION}

Pattern recognition aims to classify data (patterns) based either on a priori knowledge or on statistical information extracted from the patterns. The patterns to be classified are usually groups of measurements or observations, defining points in an appropriate multidimensional space. Its ultimate goal is to optimally extract patterns based on certain conditions and to separate one class from the others. The figure 1 shows how patterns will be classified.

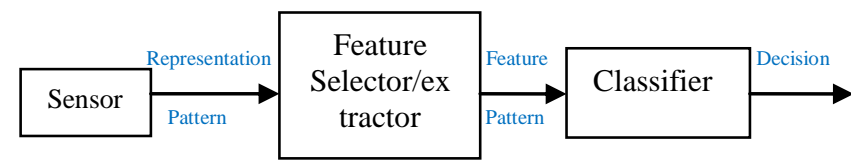

Fig 1: Pattern Classifier

SVM is a set of supervised learning method which constructs a hyperplane or set of hyperplanes in a high or infinite dimensional space, that can be used for classification, regression or other tasks. There are many hyperplanes that might classify the data, the best hyperplane is the one that represents the largest separation, or margin, between the two classes. Hence, we choose the hyperplane so that the distance from it to the nearest data point on each side is maximized. If such a hyperplane exists, it is known as the maxi Im-margin hyperplane and the linear classifier it defines is known ar a maximum margin classifier.

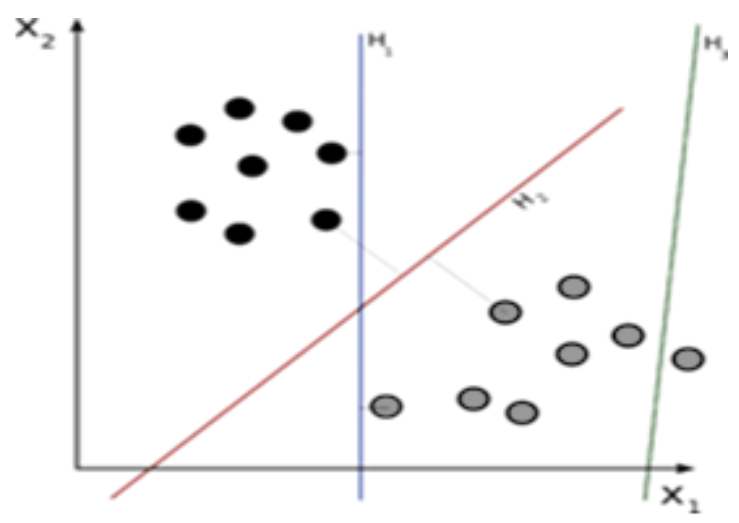

Figure 2: Maximum Margin Hyperplane

In Figure 2, H3 (green) doesn't separate the 2 classes. H1 (blue) does, with a small margin and $\mathrm{H} 2$ (red) with the maximum margin.

There are some advantages and disadvantages of support vector machines:

Advantages:

- It gives optimized result in high dimensional spaces. 
- It is memory efficient because it uses a subset of training data points in the decision function (called support vectors).

- It is versatile because different Kernel functions can be specified for the decision function. Common kernels are provided, but it is preferred to specify custom kernels.

Disadvantages:

- If the number of features of data set is much greater than the number of samples, the method is likely to give poor performances.

- SVM does not directly provide probability estimates.

\section{PROBLEM STATEMENT}

Figure 3 shows the block diagram of a novel impedanceTongue (i-Tongue). This approach uses nonspecific multielectrode electrochemical impedance spectroscopy for classification of samples of beverages in terms of sensory scores, manufacturing parameters and qualitative evaluations etc. In this approach the impedance of test solution is measured for a range of sinusoidal frequencies. The frequency specific impedance response of the electrodes is then used for classification of the samples. As a result of which a number of features come in limelight having large dimensions, so to reduce the dimension and to get optimized result feature extraction has been done by using feature extraction technique. These extracted features are then subjected to a pattern recognition engine (PARC) which classifies the sample based on its learning parameters.

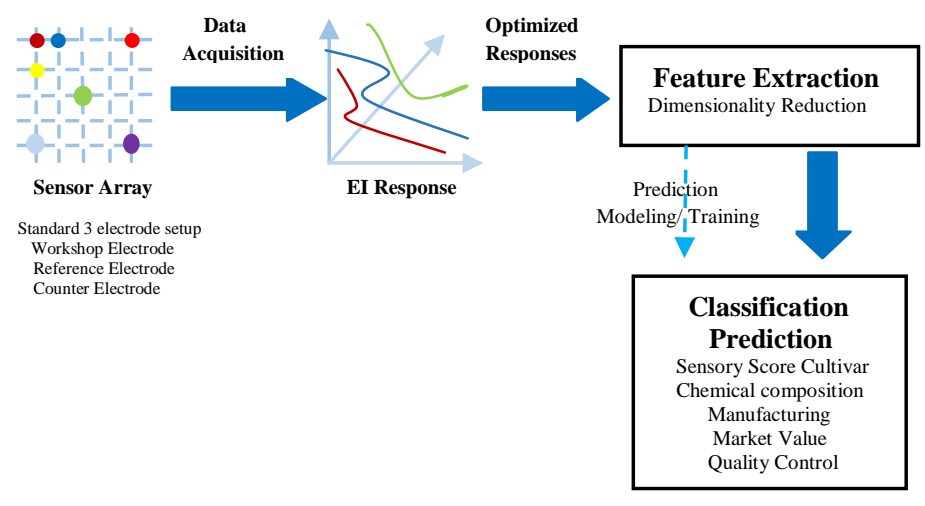

Fig 3: Generic Architecture of a novel impedance-Tongue

\subsection{Pattern Recognition Problem:}

-Formulation of the problem: a clear understanding of the aims of the investigation and planning the remaining stages.

-Data collection: making measurements on appropriate variables and recording details of the data collection procedure.

-Feature selection or feature extraction: selecting variables from the measured set that are appropriate for the task.

-Pattern classification: This may be viewed as tentative data analysis and it may provide a successful conclusion to a study.

-Apply discrimination or regression procedures as appropriate. The classifier is designed using a training set of exemplar patterns.
-Assessment of results. This may involve applying the trained classifier to an independent test set of labeled patterns.

-Interpretation.

\section{CONCLUSION AND FUTURE WORK}

We surveyed that evaluating the quality of food product, medicine, chemicals etc. is very complex task. Impedance tongue is employed for this task and maintain its capability to discriminate quality of different products. In many applications SVM is applied to recognize the pattern of the featured data samples and we found that SVM is very effective to recognize pattern and helps in differentiating the quality of different data samples.

In future work, we are going to classify different Indian tea samples by deploying SVM as classification tool followed by collecting impedance values of tea data samples exported from i-tongue. SVM is a supervised learning method in which the network is trained by providing it with input and matching output patterns.

\section{REFERENCES}

[1] B. Tudu, A. Jana, A. Metla, D. Ghosh, N. Bhattacharyya, R. Bandyopadhyay "Electronic nose for black tea quality evaluation by an incremental RBF network, Sensors and Actuators B" Chemical, Vol. 138,pp. 90-95,2009.

[2] N. Bhattacharyya, R. Bandyopadhyay, M. Bhuyan, A. Ghosh, R.K. Mudi "Correlation of multi-sensor array data with "Tasters" panel evaluation for objective assessment of black tea flavor, in" Int. Proc. ISOEN2005, Barcelona, Spain, April 13-15, 2005.

[3] A. P. Bhondekar, M. Dhiman, A. Sharma, A. Bhakta, A. Ganguli, S.S.Bari, R. Vig, P. Kapur, M. L. Singla “A novel i-Tongue for Indian black tea discrimination, CSIO Chandigarh, Sensors and Actuators B" Chemical, 2010.

[4] A. Legin, A. Rudnitskaya, D. Clapham, B. Seleznev, K. Lord and Y. Vlasov "Electronic tongue for pharmaceutical analytics - quantification of tastes and masking effects" J. Bioanalytical Chemistry, Vol 380, pp. 36-45,2004.

[5] P. Devijver and J. Kittler "Pattern Recognition: A Statistical Approach" Prentice Hall, 1982.

[6] M. Jamal, M. R. Khan, S. A. Imam "Electronic Tongue and Their Analytical Application Using Artificial Neural Network Approach: A Review" MASAUM Journal Of Reviews and Surveys, Vol. 1, Issue 1, September 2009.

[7] A. Riul, H.C. de Sousa, R.R. Malmegrim, D.S. dos Santos, A.C.P.L.F. Carvalho, F.J. Fonseca, O.N. Oliveira, L.H.C. Mattoso "Wine classification by taste sensors made from ultra-thin films and using neural networks, Sensors and Actuators B" Chemical, Vol.98, pp.77-82, 2004.

[8] N. Bhattacharyya, R. Bandyopadhyay, M. Bhuyan, A. Ghosh, R.K. Mudi, Correlation of multi-sensor array data with "Tasters" panel evaluation for objective assessment of black tea flavour, in: Int. Proc. ISOEN-2005, Barcelona, Spain, April 13-15, 2005.

[9] Yu. Vlasov, A. Legin, A. Rudnitskaya, C.DiNatale, A. D'Amico "Nonspecific sensor arrays ("electronic tongue") for chemical analysis of liquids (IUPAC Technical Report)", Pure and Applied Chemistry, Vol. 77, 1965-1983,2005. 
[10] K. Toko "Electronic sensing of tastes, Electroanalysis 10”, pp.657-669, 1998.

[11] E. Phaisangittisagul, H.T. Nagle "Sensor Selection for Machine Olfaction Based on Transient Feature Extraction, Instrumentation and Measurement", IEEE Transactions, Vol.57, No. 2, pp. 369-378, 2008.

[12] C. Tan, L. X, Z. Cao "On-Line Fuel Identification Using Optical Sensing and Support Vector Machines Technique", I2MTC 2009 - International Instrumentation and Measurement Technology Conference Singapore, 57 May, IEEE, 2009.
[13] X. Wang, H.R. Zhang, C.J. Zhang “ Signals Recognition Of Electronic Nose Based On Support Vector Machines" Proceedings of the Fourth International Conference on Machine Learning and Cybernetics, Guangzhou,18-21 August, IEEE,2005.

[14] L. Zheng and X. He "Classification Techniques in Pattern Recognition" Proceedings ISBN 80-903100-8-7 WSCG'2005, January 31-February 4, 2005. 